\title{
Bridge Engineering: Referees 2021
}

The following is a list of referees who have reviewed papers for Bridge Engineering between 17 November 2020 and 17 November 2021. The Institution of Civil Engineers is very grateful for their assistance.

We are continually looking for suitable reviewers for papers submitted to Bridge Engineering. Papers published in the Proceedings of the ICE must be submitted to at least two independent referees to judge accuracy, style, impact, importance and interest.

If you are interested in reviewing articles on any topic related to bridge engineering, please submit your name, qualifications or $\mathrm{CV}$,

Ebrahim Afsar Dizaj

Afaq Ahmad

Louis Akeh

Pere Alfaras

Sotirios Argyroudis

Qiang Bai

James Banks

Reza Barati

Elisa Bertolesi

David Bishop

Bruno Briseghella

Mark Bulmer

Simon Burchett

Colin Caprani

Alessandro Cardoni

Andrea Caristo

Nicola Cavalagli

Peter Clapham

Richard Cooke

Robert Corbally
Lee Cunningham

Alexandre Cury

Gaurav Datta

Kevin Davies

E. T. Richard Dean

Melissa Deiuliis

You Dong

João Fernandes

Tiago Ferradosa

Reza Filizadeh

Davide Forcellini

Arndt Goldack

Choa Guo

Ciaran Hanley

Chris Hendy

Stephen Hicks

Lorenzo Hofer

Junsheng Huang

Matt Joyner

Mohammad Kashani

and areas of expertise. We are in need of individuals who will agree to review papers in a timely fashion (within 3 to 4 weeks of receipt) and provide confidential feedback to the Editorial Advisory Panel concerning the quality of the paper and any suggested revisions that would be appropriate.

If you are such a person, please contact Kirsten Buchanan (tel.: +44 (0)207 665 2204; e-mail: kirsten.buchanan@icepublishing.uk) for more information on the referee process.

Ioannis Kilanitis

Yail Jimmy Kim

Michael Kotsovos

Eftychia Koursari

Alban Kuriqi

Mojtaba Labibzadeh

Mohammad Maldar

Patrick McGetrick

Ananda Medagoda Arachchige

Stergios Mitoulis

Masoud Mohammadi

Hanson Ngo

Kieran O'Connor

Vikram Pakrashi

Paul Perry

John Place

Dirk Proske

Muhammad Masood Rafi

Maria Rashidi

John Redpath
Ahlam Sader Mohammed

Meltem Saplioğlu

Vasilis Sarhosis

Hossein Shahraki

Graham Slade

William Soo Lon Wah

Nuno Sousa

Riccardo Stroscio

Ahmed Suwaed

Peter Takacs

Cem Topkaya

Georgios Tsionis

Enrico Tubaldi

Paul Vardanega

Chenghao Wang

Xin Wang

Gao Wenbo

Mahdi Yazdani

Paolo Zampieri 